\title{
Adomian Decomposition Method for Solving Delay Differential Equations of Fractional Order
}

\author{
Osama H. Mohammed and Abbas I. Khlaif \\ Al-Nahrain University, College of Science, Department of Mathematics and Computer Applications
}

\begin{abstract}
In this paper, we implement Adomian decomposition method for solving numerically non-linear delay differential equations of fractional order. The fractional derivative will be in the Caputo sense. In this approach, the solutions are found in the form of a convergent power series with easily computed components. Some numerical examples are presented to illustrate the accuracy and ability of the proposed method.
\end{abstract}

Keywords: Adomian decomposition method, delay differential equations, fractional calculus, fractional delay differential equations.

\section{Introduction}

The subject of fractional calculus (that is calculus of integrals and derivatives of any arbitrary real or complex order) has gained considerable popularity and importance during the past three decades or so, due mainly to its demonstrated applications in numerous seemingly diverse and widespread fields of science and engineering. It does indeed provide several potentially useful tools for solving differential and integral equations, and various other problem involving special functions of mathematical physics as well as their extensions and generalizations in one and more variables [1].

In real world systems, delays can be recognized everywhere and there has been widespread interest in the study of delay differential equations for many years.

However, fractional delay differential equations $\left(\mathrm{FDDE}_{\mathrm{S}}\right)$ are a very recent topic.Although it seems natural to model certain processes and systems in engineering and other science (with memory and heritage properties) with this kind of equations, only in the last few years has the attention of the scientific community been devoted to them [2].

Concerning the existence of solutions of $\left(\mathrm{FDDE}_{\mathrm{S}}\right)$ we refer [3-5]. In [3] Lakshmikantham provides sufficient conditions for the existence of solutions to initial value problems to single term nonlinear delay fractional differential equations, with the fractional derivative defined in the Riemann-Liouville sense. In [4],Yeetal. investigate the existence of positive solutions for a class of single term delay fractional differential equations.Later in [5], for the same class of equations, sufficient condition for the uniqueness of the solution are reported [2].

For the stability issues of the $\left(\mathrm{FDDE}_{\mathrm{S}}\right)$ we refer the references [6-9].

In this paper we shall use the Adomian decomposition method to find the approximate solution of the $\left(\mathrm{FDDE}_{\mathrm{S}}\right)$ with variable delays.

At the beginning of 1980 Adomian proposed new method to solve some functional equations $[10,11]$.The Adomian decomposition method has the advantage of converging to the exact solution, and can easily handle a wide class of both linear and nonlinear differential and integral equations. It decomposes the solution into the series with easily computed components which converges rapidly to the exact solution. The theoretical treatment of the convergence of the Adomian decomposition method has been considered in $[12,13,14,15]$.

\section{The structure of this paper is organized as follows:}

In section 2, we recall the definitions of fractional derivatives and fractional integration in section 3 the basic concept of the Adomian decomposition method will be given in section 4 we present our approach to solve the delay differential equation of fractional order in section 5 numerical examples are given followed by conclusions in section 6 .

\section{Fractional Derivative and Integration}

In this section we shall review the basic definitions and properties of fractional integral and derivatives, which are used further in this paper[16].

Definition(1):The Riemann-Liouville fractional integral operator of order $\alpha>0$ is defined as: 


$$
\begin{aligned}
& I_{t}^{\alpha} f(t)=\frac{1}{\Gamma(\alpha)} \int_{0}^{t}(t-x)^{\alpha-1} f(x) d x, \alpha>0, x>0 \\
& I_{t}^{0} f(t)=f(t)
\end{aligned}
$$

Definition(2):The Riemann-Liouville fractional derivative operator of order $\alpha>0$ is defined as:

$$
D_{t}^{\alpha} f(t)=\frac{1}{\Gamma(n-\alpha)} \frac{d^{n}}{d t^{n}} \int_{0}^{t}(t-x)^{n-\alpha-1} f(x) d x
$$

where $\mathrm{n}$ is an integer and $\mathrm{n}-1<\alpha \leq \mathrm{n}$.

Definition(3):The Caputo fractional derivative operator of order $\alpha$ is defined as:

where $n$ is an integer and $n-1<\alpha \leq n$.

$$
{ }^{c} D_{t}^{\alpha} f(t)=\frac{1}{\Gamma(n-\alpha)} \int_{0}^{t}(t-x)^{n-\alpha-1} \frac{d^{n}}{d t^{n}} f(t) d t
$$

Caputo fractional derivative has a useful property:

where $\mathrm{n}$ is an integer and $\mathrm{n}-1<\alpha \leq \mathrm{n}$.

$$
I_{t}^{\alpha}{ }^{c} D_{t}^{\alpha} f(t)=f(t)-\sum_{k=0}^{n-1} f^{(k)}\left(0^{+}\right) \frac{t^{k}}{k !}
$$

And similar to integer-order differentiation, Caputo fractional derivative operator is a linear operation:

$$
{ }^{c} D_{t}^{\alpha}(\lambda f(t)+\mu g(t))=\lambda{ }^{c} D_{t}^{\alpha} f(t)+\mu{ }^{c} D_{x}^{\alpha} g(t)
$$

where $\lambda$ and $\mu$ are constants. For the Caputo's derivative, also we have:

$$
{ }^{c} D_{t}^{\alpha} t^{n}=\left\{\begin{array}{c}
{ }^{c} D_{t}^{\alpha} \mathrm{C}=0, C \text { is constant } \\
0, \text { for } n \in N_{0} \text { and } n \geq[\alpha] \\
\frac{\Gamma(n+1)}{\Gamma(n+1-\alpha)} t^{n-\alpha} \text { for } n \in N \text { and } n \geq[\alpha]
\end{array}\right.
$$

We use the ceiling function $[\alpha]$ to denote the smallest integer greater than or equal to $\alpha$ and $N=\{0,1,2,3, \ldots\}$.

\section{The Adomian Decomposition Method (ADM)}

To introduce the basic idea of the ADM, we consider the operator equation $\mathrm{Fy}=\mathrm{G}$, where $\mathrm{F}$ represents a general nonlinear ordinary differential operator and $\mathrm{G}$ is a given function. Then $\mathrm{F}$ can be decomposed as:

$$
\mathrm{Ly}+\mathrm{Ry}+\mathrm{Ny}=\mathrm{G} \text { (1) }
$$

whereN is a nonlinear operator, $\mathrm{L}$ is the highest-order derivative which is assumed to be invertible, $\mathrm{R}$ is a linear differential operator of order less than $\mathrm{L}$ and $\mathrm{G}$ is the nonhomogeneous term.

The method is based by applying the operator $\mathrm{L}^{-1}$ formally to the expression:

so by using the given conditions, we obtain:

$$
\mathrm{Ly}=\mathrm{G}-\mathrm{Ry}-\mathrm{Ny}
$$

$$
\mathrm{y}=\mathrm{h}+\mathrm{L}^{-1} \mathrm{G}-\mathrm{L}^{-1} \mathrm{Ry}-\mathrm{L}^{-1} \mathrm{Ny}(3)
$$

where $h$ is the solution of the homogeneous equation $L y=0$, with the initial-boundary conditions. The problem now is the decomposition of the nonlinear term Ny. To do this, Adomian developed a very elegant technique as follows:

Define the decomposition parameter $\lambda$ as:

$$
y=\sum_{n=0}^{\infty} \lambda^{n} y_{n}
$$

then $\mathrm{N}(\mathrm{y})$ will be a function of $\lambda, \mathrm{y}_{0}, \mathrm{y}_{1} \ldots$ Next expanding $\mathrm{N}(\mathrm{y})$ in Maclurian series with respect to $\lambda$ we obtain $\mathrm{N}(\mathrm{y})=\sum_{\mathrm{n}=0}^{\infty} \lambda^{\mathrm{n}} \mathrm{A}_{\mathrm{n}}$, where:

$$
\mathrm{A}_{\mathrm{n}}=\frac{1}{\mathrm{n} !} \frac{\mathrm{d}^{\mathrm{n}}}{\mathrm{d} \lambda^{\mathrm{n}}}\left[\mathrm{N}\left(\sum_{\mathrm{k}=0}^{\mathrm{n}} \lambda^{\mathrm{k}} \mathrm{y}_{\mathrm{k}}\right)\right]_{\lambda=0}
$$

where the components of $A_{n}$ are the so called Adomian polynomials they are generated for each nonlinearity, for example, for $\mathrm{N}(\mathrm{y})=\mathrm{f}(\mathrm{y})$ the Adomian polynomials, are given as:

$$
\begin{aligned}
& \mathrm{A}_{0}=\mathrm{f}\left(\mathrm{y}_{0}\right) \\
& \mathrm{A}_{1}=\mathrm{y}_{1} \mathrm{f}^{\prime}\left(\mathrm{y}_{0}\right)
\end{aligned}
$$

$$
\begin{gathered}
A_{2}=y_{2} f^{\prime}\left(y_{0}\right)+\frac{y_{1}^{2}}{2} f^{\prime \prime}\left(y_{0}\right) \\
A_{3}=y_{3} f^{\prime}\left(y_{0}\right)+y_{1} y_{2} f^{\prime \prime}\left(y_{0}\right)+\frac{y_{1}^{3}}{3 !} f^{\prime \prime \prime}\left(y_{0}\right)
\end{gathered}
$$


Now, we parameterize eq.(3) in the form:

$$
\mathrm{y}=\mathrm{h}+\mathrm{L}^{-1} \mathrm{G}-\lambda \mathrm{L}^{-1} \mathrm{Ry}-\lambda \mathrm{L}^{-1} \mathrm{Ny}
$$

where $\lambda$ is just an identifier for collecting terms in a suitable way such that $\mathrm{y}_{\mathrm{n}}$ depends on $\mathrm{y}_{0}, \mathrm{y}_{1}, \ldots, \mathrm{y}_{\mathrm{n}}$ and we will later set $\lambda=1$.

$$
\sum_{n=0}^{\infty} \lambda^{n} y_{n}=h+L^{-1} G-\lambda L^{-1} R \sum_{n=0}^{\infty} \lambda^{n} y_{n}-\lambda L^{-1} \sum_{n=0}^{\infty} \lambda^{n} A_{n}(6)
$$

Equating the coefficients of equal powers of $\lambda$, we obtain:

and in general:

$$
\left.\begin{array}{l}
\mathrm{y}_{0}=\mathrm{h}+\mathrm{L}^{-1} \mathrm{G} \\
\mathrm{y}_{1}=-\mathrm{L}^{-1}\left(\mathrm{Ry}_{0}\right)-\mathrm{L}^{-1}\left(\mathrm{~A}_{0}\right) \\
\mathrm{y}_{2}=-\mathrm{L}^{-1}\left(\mathrm{Ry}_{1}\right)-\mathrm{L}^{-1}\left(\mathrm{~A}_{1}\right) \\
\quad \vdots
\end{array}\right\}
$$

Finally, an $\mathrm{N}$-terms that approximate solution is given by:

$$
\mathrm{y}_{\mathrm{n}}=-\mathrm{L}^{-1}\left(\mathrm{Ry_{n } - 1}\right)-\mathrm{L}^{-1}\left(\mathrm{~A}_{\mathrm{n}-1}\right), \mathrm{n} \geq 1
$$

$$
\Phi_{\mathrm{N}}(\mathrm{T})=\sum_{\mathrm{n}=0}^{\mathrm{N}-1} \mathrm{y}_{\mathrm{n}}(\mathrm{T}), \quad \mathrm{N} \geq 1
$$

and the exact solution is $y(t)=\lim _{N \rightarrow \infty} \Phi_{N}(t)$.

\section{The Approach}

In this section we shall approximate the solution of the following FDDE:

$$
\begin{gathered}
{ }^{c} D_{t}^{\alpha} y(t)=N(t, y(t), y(\Phi(t)) \\
y(t)=\psi(t),-\tau \leq t \leq 0 \\
y^{i}(0)=y_{0}^{i} i=0,1, \ldots, n-1
\end{gathered}
$$

where $\mathrm{n}-1<\alpha \leq \mathrm{n}$.

Operating $\mathrm{I}_{\mathrm{t}}^{\alpha}$ to the both sides of eq.(8), we have:

$$
\mathrm{y}(\mathrm{t})=\mathrm{I}_{\mathrm{t}}^{\alpha} \mathrm{N}(\mathrm{t}, \mathrm{y}(\mathrm{t}), \mathrm{y}(\Phi(\mathrm{t})))+\sum_{\mathrm{k}=0}^{\mathrm{n}-1} \mathrm{y}\left(0^{+}\right) \frac{\mathrm{t}^{\mathrm{k}}}{\mathrm{k} !}
$$

Adomian's method defined the solution $\mathrm{y}(\mathrm{t})$ by the series:

$$
y(t)=\sum_{n=0}^{\infty} y_{n}(t)
$$

So that, the Components $\mathrm{y}_{\mathrm{n}}$ will be determined recursively. Moreover,the method defines the nonlinear term $\mathrm{N}(\mathrm{t}, \mathrm{y}(\mathrm{t}), \mathrm{y}(\phi(\mathrm{t}))$ by the Adomian polynomials:

$$
N(t, y(t), y(\phi(t)))=\sum_{n=0}^{\infty} A_{n}
$$

where $A_{n}$ are Adomian polynomials that can be generated for all forms of nonlinearity as:

Substituting eqs.(11) and (12) into eq. (10) gives:

$$
A_{n}=\frac{1}{n !} \frac{d^{n}}{d \lambda^{n}}\left[N\left(t, \sum_{j=0}^{\infty} \lambda^{j} y_{j}(t), \sum_{j=0}^{\infty} \lambda^{j} y_{j}(\phi(t))\right)\right]_{\lambda=0}
$$

$$
\sum_{\mathrm{n}=0}^{\infty} \mathrm{y}_{\mathrm{n}}(\mathrm{t})=\sum_{\mathrm{k}=0}^{\mathrm{n}-1} \mathrm{y}^{(\mathrm{k})}\left(0^{+}\right) \frac{\mathrm{t}^{\mathrm{k}}}{\mathrm{k} !}+\mathrm{I}_{\mathrm{t}}^{\alpha}\left(\sum_{\mathrm{n}=0}^{\infty} \mathrm{A}_{\mathrm{n}}\right)
$$

To determine the componentsy $\mathrm{y}_{\mathrm{n}}(\mathrm{x}), \mathrm{n} \geq 0$. First we identify the zero component $\mathrm{y}_{0}(\mathrm{x})$ by the terms $\sum_{\mathrm{k}=0}^{\mathrm{n}-1} \mathrm{y}^{(\mathrm{k})}\left(0^{+}\right)$and $\psi(\mathrm{t})$ and $\mathrm{I}_{\mathrm{t}}^{\alpha} \mathrm{f}(\mathrm{t})$ where $\mathrm{f}(\mathrm{t})$ represents the non-homogenous term of $\mathrm{N}(\mathrm{t}, \mathrm{y}(\mathrm{t}), \mathrm{y}(\phi(\mathrm{t})))$.Second, the remaining components ofy $(\mathrm{x})$ can be determined in a way such that each component is determined by using the preceding components.In other words, the method introduces the recursive relation:

$$
\begin{aligned}
y_{0}(t)= & \psi(t)+\sum_{k=0}^{n-1} y\left(0^{+}\right) \frac{t^{k}}{k !}+I_{t}^{\alpha} f(t) \\
& y_{n+1}(t)=I_{t}^{\alpha} A_{n}, \quad n \geq 0 \quad(16)
\end{aligned}
$$

\section{Numerical Examples}

In this section we shall use the ADM to solve the non-linear fractional differential equations with delay and the results obtained using this scheme will be compare with the analytical solution

Example (1): Consider the FDDES:

$$
\begin{aligned}
{ }^{c} D_{t}^{\alpha} y(t)=\frac{1}{2} e^{\frac{t}{2}} y\left(\frac{t}{2}\right)+\frac{1}{2} y(t), 0 & \leq t \leq 1,0<\alpha \leq 1 \\
y(o) & =1
\end{aligned}
$$

The exact solution of equation (17) when $\alpha=1$ was given in [17] as $y(t)=e^{t}$. According to equations (15) and (16), thus we have: 


$$
\begin{gathered}
y_{0}(t)=1 \\
y_{n+1}(t)=I_{t}^{\alpha}\left(\frac{1}{2} e^{\frac{t}{2}} y_{n}\left(\frac{t}{2}\right)+\frac{1}{2} y_{n}(t)\right)
\end{gathered}
$$

And upon taking the Maclurian series expansion to $\mathrm{e}^{\frac{\mathrm{t}}{2}}$ up to three terms . One can get $\mathrm{y}_{1}, \mathrm{y}_{2}, \mathrm{y}_{3}, \ldots$, respectively. Following Table (1) represent the approximate solution of example (1) using ADM up to three terms for different values of $\alpha$ with a comparison with the exact solution when $\alpha=1$.

Table (1) The approximate solution of example (1) using different values of $\alpha$ with a comparison with the exact solution when $\alpha=1$.

\begin{tabular}{|c|c|c|c|c|}
\hline $\mathbf{t}$ & $\begin{array}{c}\text { ADM } \\
\boldsymbol{\alpha = 0 . 5}\end{array}$ & $\begin{array}{c}\text { ADM } \\
\boldsymbol{\alpha = 0 . 7 5}\end{array}$ & $\begin{array}{c}\text { ADM } \\
\boldsymbol{\alpha = 1}\end{array}$ & Exact \\
\hline 0 & 1 & 1 & 1 & 1 \\
\hline 0.1 & 1.45 & 1.216 & 1.105 & 1.105 \\
\hline 0.2 & 1.701 & 1.391 & 1.221 & 1.221 \\
\hline 0.3 & 1.926 & 1.565 & 1.347 & 1.35 \\
\hline 0.4 & 2.142 & 1.745 & 1.485 & 1.492 \\
\hline 0.5 & 2.356 & 1.932 & 1.636 & 1.649 \\
\hline 0.6 & 2.573 & 2.128 & 1.799 & 2.014 \\
\hline 0.7 & 2.794 & 2.334 & 1.976 & 2.226 \\
\hline 0.8 & 3.022 & 2.553 & 2.167 & 2.46 \\
\hline 0.9 & 3.26 & 2.784 & 2.374 & 2.718 \\
\hline 1 & 3.508 & 3.029 & 2.598 & \\
\hline
\end{tabular}

Example (2): Consider the FDDES:

$$
\begin{gathered}
{ }^{c} D_{t}^{\alpha} y(t)=\frac{3}{4} y(t)+y\left(\frac{t}{2}\right)-t^{2}+2,0 \leq t \leq 1,1<\alpha \leq 2 \\
y(0)=0
\end{gathered}
$$

The exact solution of equation (17) when $\alpha=2$ was given in [17] as $y(t)=t^{2}$. According to equations (15) and (16), thus we have:

$$
\begin{aligned}
y_{0}(t) & =\frac{2}{\Gamma(\alpha+1)} t^{\alpha}-\frac{2}{\Gamma(\alpha+3)} t^{\alpha+2} \\
y_{n+1}(t) & =I_{t}^{\alpha}\left(\frac{3}{4} y_{n}(t)+y_{n}\left(\frac{t}{2}\right)\right)
\end{aligned}
$$

Following Table(2) represent the approximate solution of example (2) using ADM up to three terms for different values of $\alpha$ with a comparison with the exact solution when $\alpha=2$.

Table (2) The approximate solution of example (2) using different values of $\alpha$ with a comparison with the exact solution when $\alpha=2$.

\begin{tabular}{|c|c|c|c|c|}
\hline $\mathbf{t}$ & $\begin{array}{c}\text { ADM } \\
\boldsymbol{\alpha = 1 . 5}\end{array}$ & $\begin{array}{c}\text { ADM } \\
\boldsymbol{\alpha = 1 . 7 5}\end{array}$ & $\begin{array}{c}\text { ADM } \\
\mathbf{\alpha = 2}\end{array}$ & Exact \\
\hline 0 & 0 & 0 & 0 & 0 \\
\hline 0.1 & 0.048 & 0.022 & 0.01 & 0.01 \\
\hline 0.2 & 0.137 & 0.075 & 0.04 & 0.04 \\
\hline 0.3 & 0.255 & 0.153 & 0.09 & 0.09 \\
\hline 0.4 & 0.398 & 0.254 & 0.16 & 0.16 \\
\hline 0.5 & 0.564 & 0.377 & 0.25 & 0.25 \\
\hline 0.6 & 0.753 & 0.521 & 0.36 & 0.36 \\
\hline 0.7 & 0.963 & 0.687 & 0.49 & 0.49 \\
\hline 0.8 & 1.195 & 0.873 & 0.64 & 0.64 \\
\hline 0.9 & 1.1448 & 1.08 & 0.809 & 0.81 \\
\hline 1 & 1.723 & 1.307 & 0.999 & 1 \\
\hline
\end{tabular}

Example (3): Consider the FDDES:

$$
\begin{gathered}
{ }^{c} D_{t}^{\alpha} y(t)=1-2 y^{2}\left(\frac{t}{2}\right), 0 \leq t \leq 1,0<\alpha \leq 1 \\
y(0)=0
\end{gathered}
$$

The exact solution of equation (19) when $\alpha=1$ was given in [17] as $y(t)=\sin t$.

According to eqs. (15) and (16) thus we have:

$$
\begin{gathered}
y_{0}(t)=\frac{1}{\Gamma(\alpha+1)} t^{\alpha} \\
y_{n+1}(t)=-2 I_{t}^{\alpha} A_{n}
\end{gathered}
$$

where $\mathrm{A}_{\mathrm{n}}, \mathrm{n} \geq 0$ are Adomian polynomials that represent the nonlinear term. We list the set of Adomian polynomial as: 


$$
\begin{gathered}
A_{0}(t)=y_{0}^{2}\left(\frac{t}{2}\right), \quad A_{1}(t)=2 y_{0}\left(\frac{t}{2}\right) y_{1}\left(\frac{t}{2}\right) \\
A_{2}(t)=y_{1}^{2}\left(\frac{t}{2}\right)+2 y_{0}\left(\frac{t}{2}\right) y_{2}\left(\frac{t}{2}\right) \\
A_{3}(t)=2 y_{1}\left(\frac{t}{2}\right) y_{2}\left(\frac{t}{2}\right)+2 y_{0}\left(\frac{t}{2}\right) y_{3}\left(\frac{t}{2}\right)
\end{gathered}
$$

Following Table(3) represent the approximate solution of example (3) using ADM up to three terms for different values of $\alpha$ with a comparison with the exact solution when $\alpha=1$.

Table (3) The approximate solution of example (3) using different values of $\alpha$ with a comparison with the exact solution when $\alpha=1$.

\begin{tabular}{|c|c|c|c|c|}
\hline \multicolumn{1}{|c|}{$\mathbf{t}$} & $\begin{array}{c}\text { ADM } \\
\boldsymbol{\alpha}=\mathbf{0 . 5}\end{array}$ & $\begin{array}{c}\text { ADM } \\
\boldsymbol{\alpha}=\mathbf{0 . 7 5}\end{array}$ & $\begin{array}{c}\text { ADM } \\
\boldsymbol{\alpha}=\mathbf{1}\end{array}$ & Exact \\
\hline 0 & 0 & 0 & 0 & 0 \\
\hline 0.1 & 0.329 & 0.191 & 0.1 & 0.1 \\
\hline 0.2 & 0.431 & 0.314 & 0.199 & 0.199 \\
\hline 0.3 & 0.493 & 0.413 & 0.296 & 0.296 \\
\hline 0.4 & 0.537 & 0.494 & 0.389 & 0.389 \\
\hline 0.5 & 0.574 & 0.562 & 0.479 & 0.479 \\
\hline 0.6 & 0.61 & 0.616 & 0.565 & 0.565 \\
\hline 0.7 & 0.65 & 0.66 & 0.644 & 0.644 \\
\hline 0.8 & 0.696 & 0.693 & 0.717 & 0.717 \\
\hline 0.9 & 0.752 & 0.719 & 0.783 & 0.783 \\
\hline 1 & 0.821 & 0.737 & 0.842 & 0.841 \\
\hline
\end{tabular}

\section{Conclusions}

In this paper we have been used the ADM for solving variable order delay differential equations of fractional order. Three examples were solved in the view of the ADM with good approximation and agreement with the exact solution. The results presented in this paper shows that this method gave us rapidly and acceptable solution.

\section{References}

[1] KilbasA.A., Srivastara H.M. and TrujilloJ.J., "Theory and applications of Fractional Differential Equations", ELSEVIER 2006.

[2] MoragdoM.L., Ford N. J. and Lima P.M.,"Analysis and numerical methods for fractional differential equations with delay", Journal of computational and applied mathematics 252(2013) 159-168.

[3] LakshmikanthamV., "Theory of fractional functional differential equations", J.NonlinearSci. 69 (2008) 3337-3343.

[4] Ye H., Ding Y. andGao.J.,"Theexistence of positive solution of $D^{\alpha}[x(t)-x(0)]=x(t) f(t, x t) "$, positivity 11(2007) $341-350$.

[5] Liao C. and Ye H., "Existence of positive solutions of nonlinear fractional delay differential equations",positivity 13 (2009) 601609.

[6] Chen Y. and Moore K.L., "Analytical stability bound for a class of delayed fractional-order dynamic systems", Nonlinear Dynam. 29(2002) 191-200.

[7] MihailoP.Lazarevi'c and Aleksandar M.Spasi'c, "Finite time stability analysis of fractional order time - delay systems:Gronwall's approach", Math. Comput. Modeling 49 (2009)475-481.

[8] Krolk, "Asymptotic properties of fractional delay differential equations", Appl. Math.Comput. 218(5)(2011)1515-1532.

[9] Deng W.L. C. and Lu J., "Stability of linear fractional differential system with multiple time delays", Nonlinear Dynam. 48 (2007) 409-416.

[10] Adomain G., "A review of the decomposition method and some recent results for nonlinear equation", Math. Comput.Model. 13 (7) (1992) 17.

[11] AdomianG., "Solving Frontier Problems of Physics: The Decomposition method", Kluwer, Boston, MA. 1994.

[12] AbbaouiK., CherruaultY.,"New ideas for proving convergence of decomposition methods", Comput. Math. Appl.29 (1995) 103108 .

[13] Adomain G.,Rach R.," Noise terms in decomposition series solution",Comput. Math. Appl. 24 (11) (1992) 61.

[14] Babolian B. andBiazar J.,"On the order of convergence of Adomian method", Appl. Math. Comput. 130 (2002) $383-387$.

[15] Cherruault Y., Adomian G., Abbaoui K. andRachR.,"Further remarks on convergence of decomposition method",Bio-Med. Comput. 38 (1995) 89-93.

[16] PodlubnyI.,"Fractional differential equations ", Academic Press, San Diego, 1999.

[17] D.J.Evans and K.R.Raslan, "The Adomian Decomposition Method For solving Delay Differential Equations", International Journal of Computer Mathematics, pp:1-6 (2004). 